\title{
MPLNET V3 CLOUD AND PLANETARY BOUNDARY LAYER DETECTION
}

\author{
Jasper R. Lewis ${ }^{1,2 *}$, Ellsworth J. Welton ${ }^{2}$, James R. Campbell ${ }^{3}$, Phillip C. Haftings ${ }^{2,4}$ \\ ${ }^{1}$ Joint Center for Earth Systems Technology, University of Maryland Baltimore County, Baltimore, MD \\ 21250, USA, *Email: jasper.r.lewis@nasa.gov \\ ${ }^{2}$ NASA Goddard Space Flight Center, Greenbelt, MD 20771, USA \\ ${ }^{3}$ Naval Research Laboratory, Monterey, CA 93943, USA \\ ${ }^{4}$ Science System and Applications, Inc. Lanham, MD 20706, USA
}

\begin{abstract}
The NASA Micropulse Lidar Network Version 3 algorithms for planetary boundary layer and cloud detection are described and differences relative to the previous Version 2 algorithms are highlighted. A year of data from the Goddard Space Flight Center site in Greenbelt, MD consisting of diurnal and seasonal trends is used to demonstrate the results. Both the planetary boundary layer and cloud algorithms show significant improvement of the previous version.
\end{abstract}

\section{INTRODUCTION}

The National Aeronautics and Space Administration (NASA) Micropulse Lidar Network (MPLNET) (Welton et al. 2001, http://mplnet.gsfc.nasa.gov) is a federated network of micropulse lidar (MPL) systems deployed worldwide in support of basic science and the NASA Earth Observing System program (Wielicki et al. 1995). Vertical profiles of aerosols and clouds are measured continuously both day and night at polar, midlatitude, and tropical sites using a standardized instrument and data processing algorithm. Thorough uncertainty characterization makes for straightforward comparisons between sites.

Long-tem, continuous measurements of the planetary boundary layer (PBL) depth are rare, but necessary for ascertaining the diurnal and seasonal variation for studies of air quality, weather, and climate. Similarly, it is important to have highly accurate, quantitative data records of cloud properties that span several years and geographic regions. Ground-based lidar measurements, like those provided by MPLNET, are crucial for verification and validation of satellite missions, as well as modeling processes.

\section{ALGORITHM DESCRIPTIONS}

The raw MPL signal is processed to produce the Level 1 normalized relative backscatter (NRB) which is a function of atmospheric parameters and only one instrument parameter, the lidar calibration constant. The NRB is reported at oneminute temporal resolution up to $30-\mathrm{km}$, typically at $75-\mathrm{m}$ vertical resolution (although some sites operate at $30-\mathrm{m}$ vertical resolution). The NRB is defined as:

$$
\operatorname{NRB}(z)=C \beta(z) T^{2}(z)
$$

where $C$ is the calibration constant, $\beta$ is the backscatter coefficient from molecules and particles, $T^{2}$ is the atmospheric two-way transmittance from molecules and particles, and $z$ is the altitude. The NRB and the calculation of its uncertainty are discussed by Campbell et al. (2002) and Welton and Campbell (2002). Higher level MPLNET products, including PBL and cloud retrievals, are derived from the Level 1 NRB.

\subsection{PBL Retrievals}

The Version 2 (V2) PBL depth is retrieved using a the wavelet covariance transform and a Haar wavelet as described by Davis et al. (2000) and Brooks (2003). PBL depths are determined using five-minute averages and regridded to a one-minute temporal grid. The V2 PBL products were plagued with contamination from low-level (less than $5 \mathrm{~km}$ altitude) clouds and residual layers which resulted in an unrealistic representation of the PBL.

The Version 3 (V3) PBL retrieval uses the procedure outlined by Lewis et al. (2013). The new algorithm uses the first derivative of a Gaussian wavelet in place of the Haar wavelet because it more closely resembles the gradient in 
the lidar profile and therefore results in less noise in the retrieval. Three features representing possible choices for the PBL depth are retrieved using Canny edge detection from the wavelet covariance transform. A fuzzy logic scheme is used as a means for layer attribution to select the most appropriate choice of PBL depth from the three possible features. The choice of PBL depth depends on a combination of factors including: the time of day, altitude of the retrieved feature, variance in the lidar profile, and most recently retrieved features. A continuity scheme is used to suppress unphysical fluctuations in the retrieved PBL depth.

In addition to the best estimate of the PBL depth, the altitudes of all three retrieved features are also included in the PBL products as they can be useful for identification of aerosol layers and the residual layer. Data flags are also provided to indicate day and night retrievals.

Lewis et al. (2013) demonstrated the V3 method of PBL retrieval produced shallower PBL depths which better exhibited the diurnal growth and collapse expected (see Figure 1).
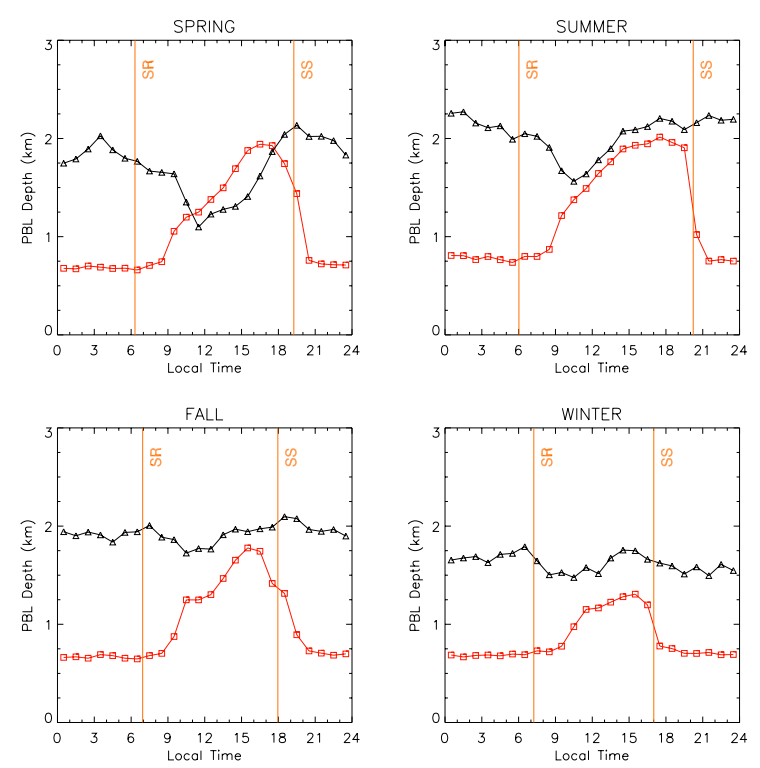

Figure 1. Comparison of V2 (black) and V3 (red) PBL depths at GSFC in 2010 (from Lewis et al. 2013)

\subsection{Cloud Retrievals}

The V2 cloud retrievals are obtained using a combination of two methods; one relying on gradients in the lidar backscatter profile, and the other based on uncertainty in the lidar signal. The first method is best suited for detection of lowlevel (primarily liquid phase) clouds and is referred to as the gradient-based cloud detection method (GCDM). The second method is designed for use in situations of low signal to noise (high solar background) and best suited for elevated clouds (i.e. cirrus). This second method is referred to as the uncertainty-based cloud detection method (UCDM). The UCDM was initially introduced by Campbell et al. (2008) to detect polar stratospheric clouds at the South Pole and uses two tunable thresholds and one objective threshold in order to resolve cloud boundaries.

The V3 cloud algorithm is based on the V2 algorithm with several meaningful changes described by Lewis et al. (2015, in preparation). The GCDM and UCDM are used in the V3 algorithm; however, a more synergistic merger of the two algorithms is used which allows the best suited method of cloud detection to be used in both day and night measurements. Previously, the UCDM was used primarily for daytime measurements retrievals when the signal-to-noise ratio was lowest.

The objective threshold used in the V3 cloud detection algorithm is attenuated within cloud layers which allows for possible better retrievals of the cloud top height and the detection of some cloud layers missed by the V2 algorithm.

The V3 cloud detection algorithm also employs a multi-temporal resolution averaging scheme to improve retrievals in conditions with high solar background. Cloud retrievals at the base one-minute temporal resolution are combined with retrievals at intermediate (fiveminute) and long (twenty-minute) averages to produce a combined cloud scene.

Cloud phase is determined using a cloud top temperature threshold suggested by Sassen and Campbell (2001) and Campbell et al. (2015) in order to identify cirrus based on the ice nucleation threshold. An estimated cloud optical depth (COD) is provided for clouds identified as cirrus using the procedure described by Chew et al. (2011). The fidelity of the COD estimate is limited by the choice of extinction-to-backscatter ratio and accuracy of cloud boundaries retrieved. 
Lidar signals are unable to penetrate through optically thick clouds which causes uncertainty in the value of the cloud top. In these cases, the estimated COD will be lower than the true optical depth. Similarly, attenuation from the bottommost cloud layer leads to uncertainty in retrievals of higher clouds for lidar profiles containing multiple cloud layers. As a result, the estimated COD will be most reliable for single-layer, optically thin clouds.

Data flags are provided to indicate the method of cloud retrieval (GCDM or UCDM), beam attenuation, and the temporal resolutions used to identify cloud layers.

Figures 2-4 show comparisons of the V2 and V3 cloud retrievals. Because the majority of changes are related to the UCDM, there are only slight differences are seen with low clouds (less than $2 \mathrm{~km}$ ) and middle clouds (between 2 and 5 $\mathrm{km})$. The largest differences are seen in relation to high clouds (above 5-km).

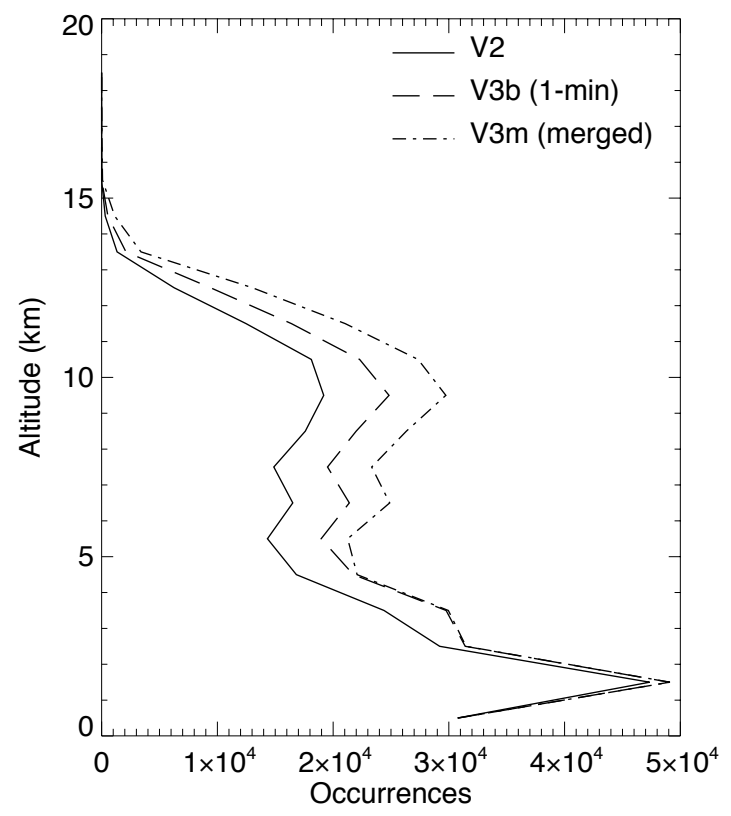

Figure 2. Vertical comparison of V2 (solid line) and V3 (dashed line and dash-dotted line for 1minute and multi-temporal resolutions, respectively) cloud retrievals.

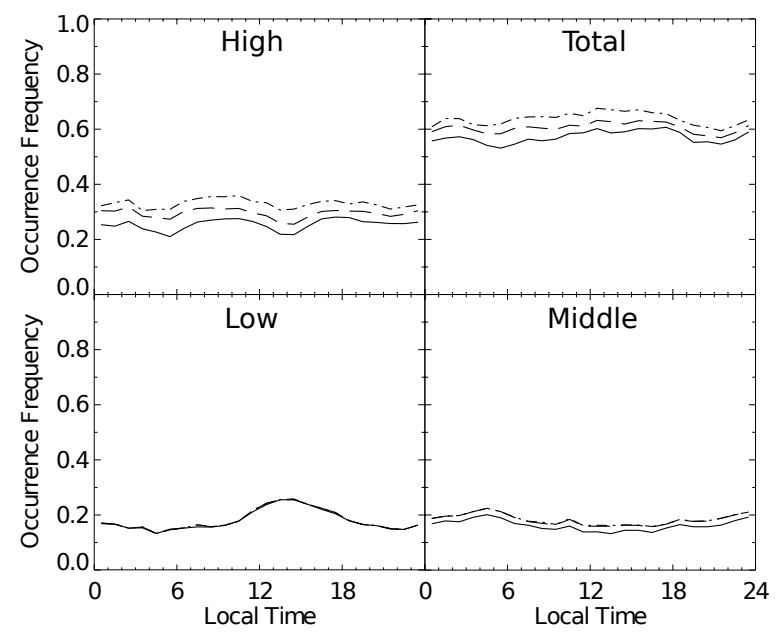

Figure 3. Diurnal comparison of V2 and V3 cloud retrievals.

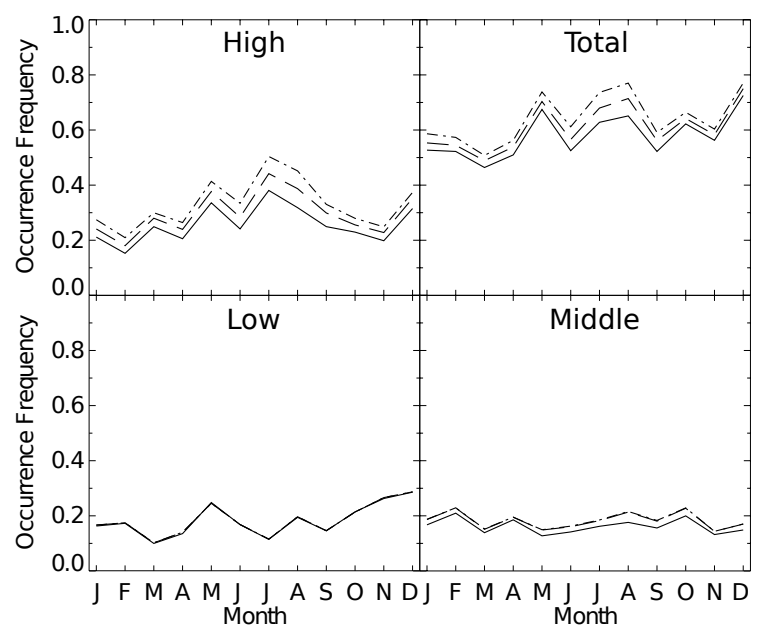

Figure 4. Monthly comparison of V2 and V3 cloud retrievals.

\section{SUMMARY}

The Version 3 planetary boundary layer and cloud algorithms are presented. The new algorithms are compared to the Version 2 algorithms to show improvements to diurnal and seasonal retrievals. Long-term continuous measurements like those provided by the Micropulse Lidar network are essential for studies of climate involving satellites and modeling processes. 


\section{ACKNOWLEDGEMENT}

We are thankful to the MPLNET PIs and staff for their efforts in establishing and maintaining the GSFC site.

\section{REFERENCES}

Campbell, J. R., D. L. Hlavka, E. J. Welton, C. J. Flynn, D. D. Turner, J. D. Spinhirne, V. S. Scott, and I. H. Hwang, 2002: Full-time, eye-safe cloud and aerosol lidar observation at Atmosphere

Radiation Measurement program sites: Instrument and data processing. J. Atmos. Oceanic Technol., 19, 431-442.

Campbell, J. R., K. Sassen, and E. J. Welton, 2008: Elevated cloud and aerosol layer retrievals from micropulse lidar signal profiles. J. Atmos. Oceanic Technol., 25, 685-700.

Campbell, J. R., M. A. Vaughan, M. Oo, R. E. Holz, J. R. Lewis, E. J. Welton, 2015: Distinguishing cirrus cloud presence in autonomous lidar measurements. Atmos. Meas. Tech., 8, 435-449, doi:10.5194/amt-8-435-2015.

Chew, B. N., J. R. Campbell, J. S. Reid, D. M. Giles, E. J. Welton, S. V. Salinas, and S. C. Liew, 2011: Tropical cirrus cloud contamination in sun photometer data. Atmos. Environ., 45, 67246731.

Lewis, J. R., E. J. Welton, A. M. Molod, and E. Joseph (2013), Improved boundary layer depth retrievals from MPLNET, J. Geophys. Res.

Atmos., 118, 9870-9879, doi:10.1002/jgrd.50570.

Sassen, K. and J. R. Campbell, 2001: A midlatitude cirrus cloud climatology from the Facility for Atmospheric Remote Sensing. Part I: Macrophysical and synoptic properties. J. Atmos. Sci., 58, 481-496.

Welton, E. J. and J. R. Campbell, 2002: Micropulse lidar signals: Uncertainty analysis. J. Atmos. Oceanic Technol., 19, 2089-2094. 